\title{
HIGHLY EFFECTIVE PHOTOTHERAPY DEVICE FOR THE TREATMENT OF JAUNDICE IN NEWBORN
}

\author{
Rijan Kafle \\ Department of Mechanical Engineering \\ New Horizon College of Engineering, \\ Bangalore, Karnataka, India
}

\begin{abstract}
Phototherapy device is intended for the treatment of neonatal unconjugated hyperbilirubinemia. All Parameters taken are anticipated to make the device portable and easy enough to handle parts and to operate the device. The use of this device does not raise new questions of safety or effectiveness for the subject device i.e. Using LED's as light source.
\end{abstract}

\section{Keywords - Phototherapy, Hyperbilirubinemia, LED's}

\section{INTRODUCTION}

Phototherapy is the use of visible light for the treatment of hyperbilirubinemia. Therapy lowers the serum bilirubin level by transforming bilirubin into water-soluble isomers that can be eliminated without conjugation in the liver. Use of LED's is one of the most recent innovations in phototherapy. These devices provide high irradiance in the blue to blue green spectrum without excessive heat generation.

The rest of the paper is organized as follows. Device effectiveness parameters and all required materials are explained in section II. Concluding remarks and experimental results are given in section III. Design concepts based on research are presented in section IV.

\section{DEVICE EFFECTIVENESS PARAMETERS AND ALL REQUIRED MATERIALS}

1. Effective treatment mode

Double-sided (under baby and over baby phototherapy).

\section{Justification:}

- Light source coverage should be at least $80 \%$ of the infant's body surface for effective therapy. So, it is not recommended to apply therapy light from only one direction.[1]

- Focused light at a particular area doesn't result in effective treatment.

2. Effective Intensity

$40 \mu \mathrm{W} / \mathrm{cm}^{2} / \mathrm{nm} \pm 25 \%$
- There may be a saturation point at $30 \mu \mathrm{W} / \mathrm{cm}^{2} / \mathrm{nm}$ where an increase in irradiance has no increased benefit in decreasing bilirubin levels.[2]

3. Human safety factor with respect to irradiance

- This level light intensity does not raise new questions of safety or effectiveness for the subject device i.e. Using LED's as light source.

4. Type of device recommended as per the sites of sites of use

- Home use:

Portable and easy enough to handle parts and to operate the device.

- Hospital or health care hub:

Portable free standing or Stand supported device.

5. Height (Between an infant's body and source of light)

Not greater than $50 \mathrm{~cm}$ (20 in) and can be less (down to $10 \mathrm{~cm}$ )

Distance less than 10:

Justification:

- It may result in burning of infant's skin due to high temperature.

- High risk of dry and itchy skin.

$>$ Distance more than $50 \mathrm{~cm}$ :

- Low light intensity.

- More power is required to maintain irradiance level at this distance.[3]

6. Most effective position of an infant's during therapy Lying horizontally with the face and trunk facing up. Justification:

- It results in the rapid reduction of the serum bilirubin level.

7. Device temperature

It is very much necessary to maintain normal human body temperature. i.e. $(37-38){ }^{\circ} \mathrm{C}$. 
8. Selection of Materials POLYCARBONATE

\section{Justification:}

For example, "LEXAN", turns out to be very adapted to this use because of its properties:

- Non-toxic

- Unbreakable

- Fire retardant

- Noise absorbing

- Heat resistant

- Water repellent

- Non- sensitive to oils, fats, diluted acids or normal disinfectants.

* Device/ bed: - ABS bed casing with acrylic sheet.

\section{Justification:}

- To maintain mechanical safety because it has tensile Strength of $75 \mathrm{MPa}$ and Flexural Strength of 115 $\mathrm{MPa}$.

- Because of its Optical Properties i.e. Light Transmission $>92 \%$ and Refractive Index 1.49. which allow blue light to travel through it with negligible deviance.

* Mattress (gel mat): - Clear polyurethane cover filled with polyurethane gel.

\section{Justification:}

- Clear to opaque i.e. transparent.

- Polyurethane gel products are pleasantly soft to the touch and return to their original shape after you touch them.

- Insulating.

- Natural adhesiveness. [5]

* Mattress cover: - Polypropylene fabric disposable.

Justification:

- Transparent.

- It is a waterproof fabric so it does not have any extra effect on humidity during therapy time.

- It is lightweight and extremely durable

III. RESEARCH BASED CONCLUSIONAND EXPERIMENTAL REMARKS

\section{A comparison of the effectiveness of LED phototherapy} machines:

\section{* Single and double-sided, for treating neonatal} jaundice in a low resource setting:-

Double-sided machines showed following details in comparison with Single Sided machines. (Comparisons were made in between firefly device and GE single sided device): -
- $\quad 54 \%$ increase in the hourly speed of TSB reduction.

- $\quad 45 \%$ increase in the speed of TSB reduction over the duration of treatment.

- $21 \%$ reduction in the duration of treatment

- $16 \%$ reduction of length of stay. [6]

\section{DESIGN CONCEPTS BASED ON RESEARCH}

1. Free stand supported device:

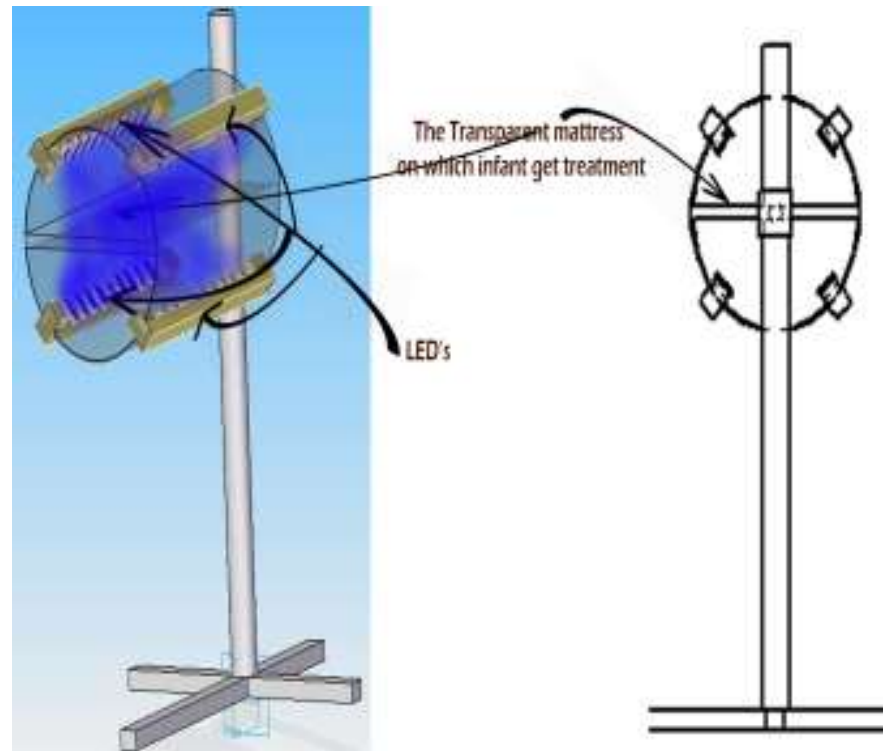

This particular free stand supported device which provides therapy light from almost all possible direction.

2. Portable device:

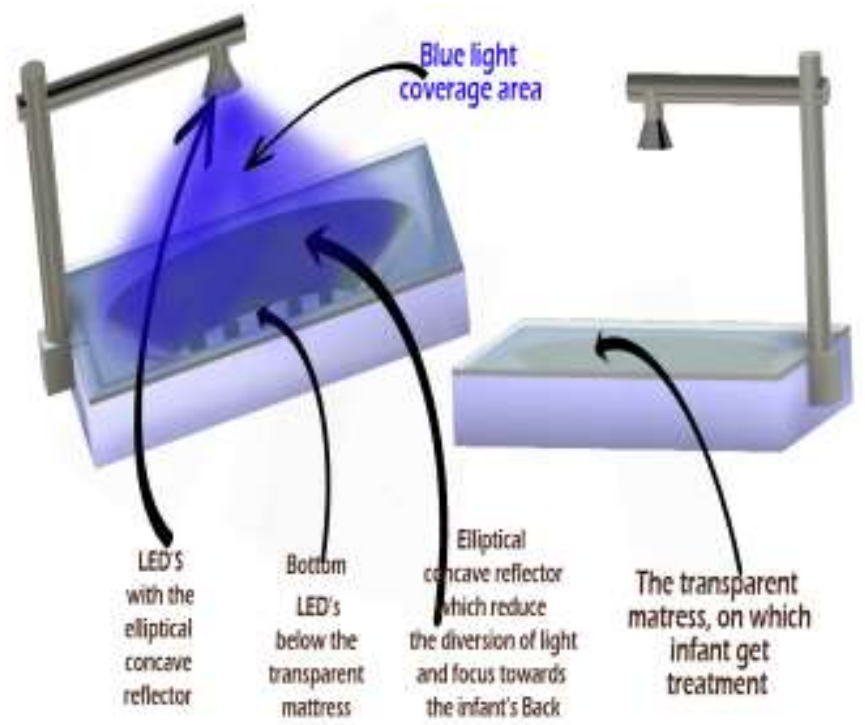




\section{International Journal of Engineering Applied Sciences and Technology, 2019 \\ Vol. 4, Issue 3, ISSN No. 2455-2143, Pages 409-411 \\ Published Online July 2019 in IJEAST (http://www.ijeast.com)}

This is a highly effective phototherapy device with maximum body coverage, compact in size and cost efficient as we are using LED's as a light source from both the sides.

3. Portable device with foldable side walls:

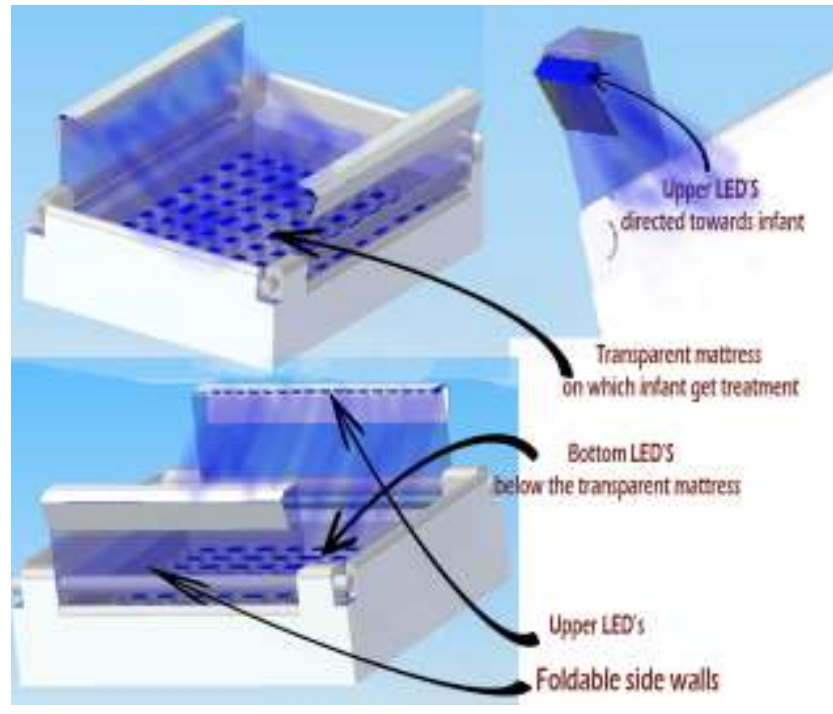

This portable device provides therapy light from almost all possible direction. And also compact in size and cost efficient as we are using LED's as a light source.

According to Mayo clinic report:

Length and head circumference dimensions by gestational age (Premature Birth)

\section{- Boys:}

Gestational age: - 32 weeks to 40 weeks Length: $-42 \mathrm{~cm}$ to $50 \mathrm{~cm}$

Head circumference: $-29 \mathrm{~cm}$ to $35 \mathrm{~cm}$

- Girls:

Gestational age: - 32 weeks to 40 weeks Length: $-42 \mathrm{~cm}$ to $51 \mathrm{~cm}$

Head circumference: $-29 \mathrm{~cm}$ to $35 \mathrm{~cm}$ [7]

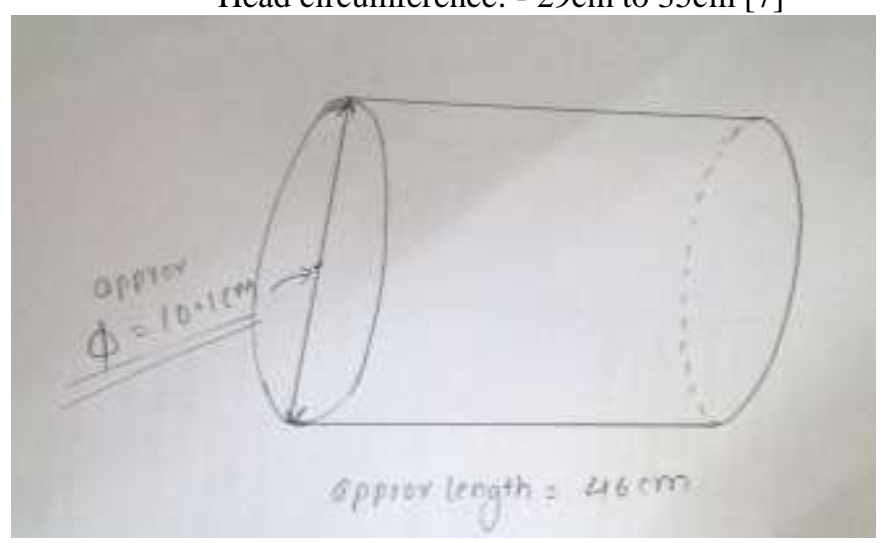

Approximate surface area of an infant $=1472 \mathrm{~cm}^{2}$ Approximate power required $=20.976 \sim 21 \mathrm{Watt}$

\section{REFERENCE}

Websites and journals Referred

[1] Laura A. Stokowski, MS, RN, Fundamentals of Phototherapy for Neonatal Jaundice,Advances in Neonatal Care, December 2006, Vol. 11, No. 5S

[2] Cremer RJ, Perryman PW, Richards DH. Influence of light on the hyperbilirubunaemia of infants. Lancet 1958; 1: 1094-7

[3] Rose Marie Rice,Shannon D. Dawson, Methods and Apparatus for Stabilizing a Subject Undergoing Phototherapy Treatment,US20080116401A1

[4] Ginevri SRL,2009-11-09, A sensible Guide to the choice of an Incubator

[5] https://www.carvilleplastics.com

[6] Renee Miller, What Is Polyurethane Fabric? www.hunker.com

[7] GE Health Care, Lullaby LED Phototherapy System, DOC1177900, JB31296INC

[8] Research article, A comparison of the effectiveness of three LED phototherapy machines, single- and doublesided, for treating neonatal jaundice in a low resource setting. Gaston Arnolda,Tran Dinh Chien,Andrew Hayen,Nguyen Thi Xuan Hoi,Katherine Maningas,Priscilla Joe,Francesco Cavallin,Daniele Trevisanuto ,Luciano Moccia, October 11, 2018. 